\title{
Glass phase and other multiple liquid-to-liquid transitions resulting from two-liquid phase competition
}

\author{
Robert F. Tournier ${ }^{1,2}$ \\ ${ }^{1}$ Univ. Grenoble Alpes, Inst. NEEL, F-38042 Grenoble Cedex 9, France \\ ${ }^{2}$ CNRS, Inst. NEEL, F-38042 Grenoble Cedex 9, France \\ E-mail address: robert.tournier@ neel.cnrs.fr
}

\begin{abstract}
Melt supercooling leads to glass formation. Liquid-to-liquid phase transitions are observed depending on thermal paths. Viscosity, density and surface tension thermal dependences measured at heating and subsequent cooling show hysteresis below a branching temperature and result from the competition of two-liquid phases separated by an enthalpy difference depending on temperature. The nucleation classical equation of these phases is completed by this enthalpy saving existing at all temperatures. The glass phase thermodynamic parameters and their thermal variation have already been determined in such a two-liquid model. They are used at high temperatures to predict liquid-to-liquid transitions in some metallic glass-forming melts.
\end{abstract}

\section{1-Introduction}

Phenomena of branching thermal properties of melts measured at heating and subsequent cooling have been observed for a long time and explained by the irreversible breakdown of a metastable microheterogeneous state of melts inherited from the original crystal sample or appearing in the process of melting [1-3]. This breakdown happens when heating up to the homogenizing temperature of the melts, which is often close to the branching temperature. The recent observations of liquid-to-liquid phase transitions (LLPT) in well-known metallic glass-forming melts raise the question of the competition between two homogeneous liquid phases numbered 1 and 2 separated by an enthalpy difference depending on the temperature [4-10]. The glass phase formation is often viewed as being due to a true thermodynamic transition. Various microscopic models and experiments prove its existence at $\mathrm{T}_{\mathrm{g}}$ [11-22]. The nucleation classical equation has been completed by introducing enthalpy savings $\varepsilon_{1 \mathrm{~s} x} \Delta \mathrm{H}_{\mathrm{m}}$ and $\varepsilon_{\mathrm{gs}} \times \Delta \mathrm{H}_{\mathrm{m}}$, respectively associated with growth nucleus formation giving rise to crystallization in Phase 1 above $\mathrm{T}_{\mathrm{g}}$ and Phase 2 below $\mathrm{T}_{\mathrm{g}}$, where $\Delta \mathrm{H}_{\mathrm{m}}$ is the melting heat per g-atom [22]. The enthalpy saving $\Delta \varepsilon_{\mathrm{lg}} \times \Delta \mathrm{H}_{\mathrm{m}}$ associated with the glass formation is then equal to $\left(\varepsilon_{\mathrm{ls}}-\varepsilon_{\mathrm{gs}}\right) \times \Delta \mathrm{H}_{\mathrm{m}}$. The energy saving coefficients $\varepsilon_{\mathrm{ls}}$ and $\varepsilon_{\mathrm{gs}}$ are linear functions of $\theta^{2}=\left(\mathrm{T}-\mathrm{T}_{\mathrm{m}}\right)^{2} / \mathrm{T}_{\mathrm{m}}{ }^{2}$, as shown by a study of supercooling rate maxima of liquid elements [23] and are equal to $\varepsilon_{1 \mathrm{~s} 0}$ and $\varepsilon_{\mathrm{gs} 0}$ at $\mathrm{T}_{\mathrm{m}}$. The minimum value 0.217 of $\varepsilon_{1 \mathrm{~s} 0}$ and $\varepsilon_{\mathrm{gs} 0}$ determined in many liquid elements at their melting temperature $\mathrm{T}_{\mathrm{m}}$ corresponds to the Lindemann coefficient 0.103 [24]. The first-order transition to an ultrastable glass of confined liquid helium under pressure has been successfully described using $\varepsilon_{1 \mathrm{~s} 0}=\varepsilon_{\mathrm{gs} 0}=0.217$ [25]. The transformation temperature of glasses in ultrastable phases having higher density has been defined as a function of an excess of frozen enthalpy $\Delta \varepsilon$. The enthalpy of the ultrastable phase attains the minimum and the density the maximum when $\Delta \varepsilon$ is equal to $\Delta \varepsilon_{\mathrm{lg} 0}=\varepsilon_{\mathrm{ls} 0}-\varepsilon_{\mathrm{gs} 0}$ at $\mathrm{T}_{\mathrm{m}}$. The time dependence of $\mathrm{T}_{\mathrm{g}}$ is explained by a weak excess enthalpy increasing $\mathrm{T}_{\mathrm{g}}$ and relaxing after cooling [26]. 
In this paper, a positive sign of $\Delta \varepsilon_{\mathrm{lg}}=\varepsilon_{\mathrm{ls}}-\varepsilon_{\mathrm{gs}}$ shows that Phase 1 is favored and a negative value that it is Phase 2. The glass phase below $\mathrm{T}_{\mathrm{g}}$ corresponds to a liquid in Phase 2. The transformation of Phase 2 in a denser phase is not included in the enthalpy changes in order to simplify the presentation. The coefficients $\varepsilon_{1 \mathrm{~s}}$ and $\varepsilon_{\mathrm{gs}}$, being functions of $\theta^{2}$, are used above $\mathrm{T}_{\mathrm{m}}$. The homogeneous nucleation temperatures $\mathrm{T}_{\mathrm{n}}$ below $\mathrm{T}_{\mathrm{m}}$ and $\mathrm{T}_{\mathrm{n}+}$ above $\mathrm{T}_{\mathrm{m}}$ of Phases 1 and 2 are solutions of the classical nucleation equation completed by the enthalpy saving $\Delta \varepsilon_{\lg } \times \Delta \mathrm{H}_{\mathrm{m}}$ and lead to first-order LLPT accompanied by latent heats equal to $\Delta \varepsilon_{\mathrm{lg}} \times \Delta \mathrm{H}_{\mathrm{m}}$. Two branching temperatures $\mathrm{T}_{\mathrm{Br}}$ of thermal properties are defined for $\Delta \varepsilon_{\mathrm{lg}}=0$. The overheating and quenching temperatures $\mathrm{T}_{\mathrm{q} 1}$ and $\mathrm{T}_{\mathrm{q} 2}$ leading to the freezing of enthalpy excesses equal to $\Delta \varepsilon_{\mathrm{lg} 0}=\varepsilon_{\mathrm{ls} 0}-\varepsilon_{\mathrm{gs} 0}$ and $2 \times \Delta \varepsilon_{\lg 0}$ are determined. The nucleation temperature of Phase 1 in the presence of an enthalpy excess $\Delta \varepsilon_{\lg 0}$ is also calculated and called $\mathrm{T}_{\mathrm{n} \text {-. }}^{\mathrm{ex}}$. The theoretical solution is attained with a superheating up to $\mathrm{T}_{\mathrm{ts}}$, where $\varepsilon_{\mathrm{gs}}$ becomes equal to zero. The enthalpy saving coefficients $\varepsilon_{\mathrm{ls}}$ and $\varepsilon_{\mathrm{gs}}$ are counted from this

$\mathrm{t}$ solution. Some of these calculated temperatures are successfully compared with various experimental determinations. The existence of a weak enthalpy excess relaxing at $\mathrm{T}_{\mathrm{g}}$ in Phase 2 inducing a time dependence of the glass transition is demonstrated.

\section{2-Basic equations}

The nucleation equation is given by (1):

$$
\Delta G=\frac{4 \pi R^{3}}{3} \Delta H_{m} / V_{m} \times(\theta-\varepsilon)+4 \pi R^{2}(1+\varepsilon) \sigma_{1} \Delta H_{m} / V_{m}
$$

where $\Delta \mathrm{G}$ is the Gibbs free energy change associated with the formation of a spherical growth nucleus of radius $\mathrm{R}, \varepsilon$ being a fraction of the melting enthalpy $\Delta \mathrm{H}_{\mathrm{m}}, \mathrm{V}_{\mathrm{m}}$ the molar volume and $\theta=\left(\mathrm{T}-\mathrm{T}_{\mathrm{m}}\right) / \mathrm{T}_{\mathrm{m}}$ the reduced temperature. The critical nucleus can give rise to crystallization or to Phase 1 or Phase 2 according to the value of the coefficient $\varepsilon$. The new surface energy is $(1+\varepsilon) \times \sigma_{1}$ instead of $\sigma_{1}$. The classical equation is obtained for $\varepsilon=0$ [27].

The homogeneous nucleation temperatures are $\theta_{\mathrm{n}-}=(\varepsilon-2) / 3$ for $\theta<0$ and $\theta_{\mathrm{n}+}=\varepsilon$ for $\theta>0$. The nucleation temperature $\theta_{n+}=\varepsilon$ for $\theta>0$ has not been used up to now. It can be found in [22 above (13)]. The coefficients $\varepsilon_{\mathrm{ls}}$ and $\varepsilon_{\mathrm{gs}}$ are values of $\varepsilon(\theta)$ leading to a nucleus formation having the critical radius for crystal growth in Phase 1 and Phase 2 respectively.

$$
\begin{aligned}
& \varepsilon_{l s}(\theta)=\varepsilon_{l s 0}\left(1-\theta^{2} \times \theta_{0 m}^{-2}\right), \\
& \varepsilon_{g s}(\theta)=\varepsilon_{g s 0}\left(1-\theta^{2} \times \theta_{0 g}^{-2}\right)+\Delta \varepsilon,
\end{aligned}
$$

where $\Delta \varepsilon$ is a coefficient of enthalpy excess which may be frozen after quenching the melt [26]. Equations (2) and (3) are respected at the homogeneous nucleation temperatures of Phase 1 and Phase 2 and (4) is 
deduced to determine the nucleation temperature $\theta_{\mathrm{n}-}$ of the glass phase called Phase 2 :

$\theta_{n-}^{2} \times \varepsilon_{g s 0} \times \theta_{0 g}^{-2}+3 \theta_{n-}+2-\varepsilon_{g s 0}-\Delta \varepsilon=0$

The solutions for $\theta_{\mathrm{n}-}$ are given by (5):

$\theta_{n-}=\left(-3 \pm\left[9-4\left(2-\varepsilon_{g s 0}-\Delta \varepsilon\right) \varepsilon_{g s 0} / \theta_{0 g}^{2}\right]^{1 / 2}\right) \theta_{0 g}^{2} /\left(2 \varepsilon_{g s 0}\right)$

The highest value of $\theta_{\mathrm{n}-}$ is chosen with sign + in (5). The glass transition reduced temperature $\theta_{\mathrm{n}-}=\theta_{\mathrm{g}}$ is

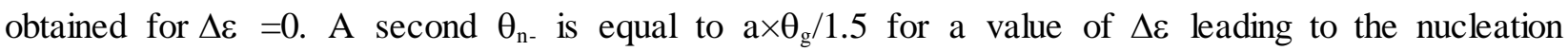
temperature of Phase 1 in Phase 2 and to the homogeneous nucleation temperature of crystallization in Phase 1 . Another value of $\theta_{\mathrm{n} \text { - }}$ equal to $\theta_{\mathrm{n} \text { - }}^{\mathrm{ex}}$ is obtained for $\Delta \varepsilon=\varepsilon_{\mathrm{ls} 0}-\varepsilon_{\mathrm{gs} 0}$ as discussed below.

An enthalpy excess $\Delta \varepsilon \times \Delta \mathrm{H}_{\mathrm{m}}$ depending on the quenching temperature can be frozen in Phase 2 by rapid cooling. Two reduced temperatures $\theta_{\mathrm{q} 1}$ and $\theta_{\mathrm{q} 2}$ of quenching leading to $\Delta \varepsilon=\left(\varepsilon_{\mathrm{ls} 0}-\varepsilon_{\mathrm{gs} 0}\right)=\Delta \varepsilon_{\lg 0}$ and to $\Delta \varepsilon$ $=2 \times \Delta \varepsilon_{\lg 0}$ are calculated. They are defined for $\Delta \varepsilon_{\lg }=0$ in (10). An overheating of Phase 1 above $\theta_{\mathrm{q} 1}$ and a subsequent slow cooling down to $\theta_{\mathrm{q} 1}$ would have to produce a new LLPT leading to Phase 2 at this temperature. The enthalpy excess coefficient $\Delta \varepsilon=\Delta \varepsilon_{\lg 0}$ is privileged because it gives rise to the most ultrastable glass phase at a vapor deposition temperature $\mathrm{T}_{\mathrm{sg}}$ having the highest enthalpy saving [26]. All other values of $\Delta \varepsilon$ smaller than $2 \times \Delta \varepsilon_{\lg 0}$ lead to deposited ultrastable phase having larger enthalpy. It is inferred that the enthalpy excess $\Delta \varepsilon_{\mathrm{lg} 0} \times \Delta \mathrm{H}_{\mathrm{m}}$ maximizing the enthalpy saving of the ultrastable glass phase is frozen in glasses that are superheated in the temperature window delimited by $\theta_{\mathrm{q} 1}$ and $\theta_{\mathrm{q} 2}$ and rapidly cooled at low temperatures.

In fragile liquids, $\theta_{0 \mathrm{~m}}$ being larger than $-2 / 3$ [22], the coefficients $\varepsilon_{1 \mathrm{~s} 0}$ and $\theta_{0 \mathrm{~m}}$ in Phase 1 are given by (6)

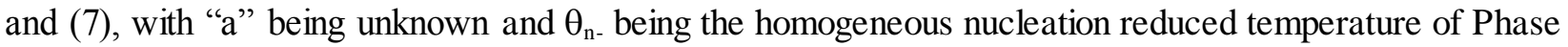
1 in Phase 2 and of growth nuclei giving rise to crystallization in Phase 1 after a very long time of relaxation:

$\varepsilon_{l s 0}=\varepsilon_{l s}(\theta=0)=1.5 \times \theta_{n-}+2=a \times \theta_{g}+2$,

where $\theta_{\mathrm{n}-}$ is equal to $\mathrm{a} \times \theta_{\mathrm{g}} / 1.5$. The coefficient $\varepsilon_{\mathrm{ls} 0}$ is maximized by (6) [28-30] .

$\theta_{0 m}^{2}=\frac{8}{9} \varepsilon_{l s 0}-\frac{4}{9} \varepsilon_{l s 0}^{2}$

The coefficients $\varepsilon_{\mathrm{gs} 0}$ and $\theta_{0 \mathrm{~g}}$ are given at $\mathrm{T}_{\mathrm{g}}$ and below $\mathrm{T}_{\mathrm{g}}$ by (8) and (9):

$$
\begin{aligned}
& \varepsilon_{g s 0}=\varepsilon_{g s}(\theta=0)=1.5 \times \theta_{g}+2, \\
& \theta_{0 g}^{2}=\frac{8}{9} \varepsilon_{g s 0}-\frac{4}{9} \varepsilon_{g s 0}^{2} .
\end{aligned}
$$


The reduced temperature $\theta_{\mathrm{g}}$ is the homogeneous nucleation reduced temperature of Phase 2 in Phase 1 and of growth nuclei giving rise to crystallization in Phase 2 after a very long time of relaxation. The coefficient $\varepsilon_{\mathrm{gs} 0}$ is also maximized by (8) [28-30].

The enthalpy saving coefficient $\Delta \varepsilon_{\mathrm{lg}}$ of Phase 1 with regard to Phase 2 is given by (10) for $\Delta \varepsilon=0$ :

$$
\Delta \varepsilon_{\mathrm{lg}}(\theta)=\left(\varepsilon_{l s}-\varepsilon_{g s}\right)=\varepsilon_{l s 0}-\varepsilon_{g s 0}+\Delta \varepsilon-\left(\theta^{2} \varepsilon_{l s 0} / \theta_{0 m}^{2}-\theta^{2} \varepsilon_{g s 0} / \theta_{0 g}^{2}\right)
$$

Phase 1 is favored when $\Delta \varepsilon_{\mathrm{lg}}>0$ and Phase 2 when $\Delta \varepsilon_{\mathrm{lg}}<0$ for $\Delta \varepsilon=0$. The phase changes occur at the homogeneous nucleation temperatures.

The reduced temperatures $\theta_{\mathrm{Br}}$ below which thermal properties are irreversible are determined for $\Delta \varepsilon_{\mathrm{lg}}=0$ in (11):

$$
\theta_{B r}= \pm\left[\left(\varepsilon_{l s 0}-\varepsilon_{g s 0}\right) /\left(\varepsilon_{l s 0} \theta_{0 m}^{-2}-\varepsilon_{g s 0} \theta_{0 g}^{-2}\right)\right]^{1 / 2}
$$

The specific heat change $\Delta \mathrm{C}_{\mathrm{p}}(\mathrm{T})$ from Phase 2 to Phase 1 is given in (12) by $\mathrm{d} \Delta \varepsilon_{\mathrm{lg}} / \mathrm{dT}$ multiplied by $\Delta \mathrm{H}_{\mathrm{m}}$ [22].

$$
\Delta C_{p}(T)=2 \frac{T-T_{m}}{T_{g}-T_{m}} \frac{\Delta H_{m}}{T_{m}}\left[\frac{9}{4 a}-\frac{9}{6}\right]
$$

The parameter "a" in (6) is determined by the specific heat jump at $\mathrm{T}_{\mathrm{g}}$ when $\mathrm{T}_{\mathrm{m}}$ and $\Delta \mathrm{H}_{\mathrm{m}}$ are known. A lot of glasses follow the scaling law (6) in which a $=1$, leading to $\Delta \mathrm{C}_{\mathrm{p}}\left(\mathrm{T}_{\mathrm{g}}\right)=1.5 \times \Delta \mathrm{H}_{\mathrm{m}} / \mathrm{T}_{\mathrm{m}}$ [22].

The homogeneous nucleation reduced temperature of Phase 2 in Phase 1 above $T_{m}$ is given by (13) respecting $\Delta \varepsilon_{\mathrm{lg}}=\theta_{\mathrm{n}+}$ :

$$
\left(\frac{\varepsilon_{l s 0}}{\theta_{0 m}^{2}}-\frac{\varepsilon_{g s 0}}{\theta_{0 g}^{2}}\right) \theta_{n+}^{2}+\theta_{n+}-\left(\varepsilon_{l s 0}-\varepsilon_{g s 0}+\Delta \varepsilon\right)=0
$$

The solution for $\theta_{\mathrm{n}+}$ is given by (14) for $\Delta \varepsilon=0$ :

$$
\theta_{n+}=\frac{-1+\left(1+4\left(\varepsilon_{l s 0}-\varepsilon_{g s 0}\right)\left(\varepsilon_{l s 0} \theta_{0 m}^{-2}-\varepsilon_{g s 0} \theta_{0 g}^{-2}\right)\right)^{1 / 2}}{2\left(\varepsilon_{l s 0} \theta_{0 m}^{-2}-\varepsilon_{g s 0} \theta_{0 g}^{-2}\right)}
$$

Phase 1 disappears when $\varepsilon_{1 \mathrm{~s}}$ in (2) becomes equal to zero. The reduced temperature $\theta_{\mathrm{ts}}$ above which all residual Phase 2 clusters cannot survive in a unique liquid and a theoretical solution of all atoms is given by (15) corresponding to $\varepsilon_{\mathrm{gs}}=0$ in (3):

$\theta_{\mathrm{ts}}=\theta_{0 \mathrm{~g}}$ 


\section{3-Application to glass-forming melts obeying scaling laws}

A broad fraction of metallic and non-metallic glasses have a specific heat jump $\Delta \mathrm{C}_{\mathrm{p}}\left(\mathrm{T}_{\mathrm{g}}\right)=1.5 \times \Delta \mathrm{S}_{\mathrm{m}}, \Delta \mathrm{S}_{\mathrm{m}}$ $=\Delta \mathrm{H}_{\mathrm{m}} / \mathrm{T}_{\mathrm{m}}$ being the melting entropy. This value corresponds to a $=1$ in $[6,12]$. The Vogel-Fulcher Tammann temperature of many polymers follows a scaling law [31] in agreement with (6). The quantity $\Delta \varepsilon_{\lg 0}$ is equal $-0.5 \times \theta_{\mathrm{g}}$. The following LLPT are expected in this family:

$$
\begin{array}{lc}
\theta_{\mathrm{n}-}=2 / 3 \times \theta_{\mathrm{g}}, & \varepsilon_{\mathrm{ls}}-\varepsilon_{\mathrm{lg}}=-0.16666 \times \theta_{\mathrm{g}}, \\
\theta_{\mathrm{n}-}^{\mathrm{ex}}=0.42222 \times \theta_{\mathrm{g}} & \varepsilon_{\mathrm{ls}}-\varepsilon_{\mathrm{lg}}=-0.3663 \times \theta_{\mathrm{g}} \\
\theta_{\mathrm{n}+}=-0.38742 \times \theta_{\mathrm{g}} & \varepsilon_{\mathrm{ls}}-\varepsilon_{\mathrm{lg}}=-0.38742 \times \theta_{\mathrm{g}} \\
\theta_{\mathrm{Br}}= \pm 0.8165 \times \theta_{\mathrm{g}} & \varepsilon_{\mathrm{ls}}-\varepsilon_{\mathrm{lg}}=0 \\
\theta_{\mathrm{q} 1}=-1.1547 \times \theta_{\mathrm{g}} & \varepsilon_{\mathrm{ls}}-\varepsilon_{\mathrm{lg}}=\varepsilon_{\mathrm{ls} 0}-\varepsilon_{\mathrm{g} s 0}=-0.5 \times \theta_{\mathrm{g}}
\end{array}
$$

The superheating temperature $\theta_{\mathrm{q} 2}=-\theta_{\mathrm{g}} \times \sqrt{2}$ corresponds to $\Delta \varepsilon=2 \times\left(\varepsilon_{1 \mathrm{~s} 0}-\varepsilon_{\mathrm{g} s 0}\right)=-\theta_{\mathrm{g}}$.

Phase 1 disappears above a reduced temperature equal to $-\theta_{0 \mathrm{~m}}=\left[\left(2+\theta_{\mathrm{g}}\right) \times\left(-4 \times \theta_{\mathrm{g}} / 9\right)\right]^{1 / 2}$ in all glass-forming melts.

Phase 2 clusters disappear above $\theta_{\mathrm{ts}}=\left[\left(1.5 \times \theta_{\mathrm{g}}+2\right) \times\left(-2 \times \theta_{\mathrm{g}} / 3\right)\right]^{1 / 2}$ in all glass-forming melts.

\section{4-Application to some metallic glass-forming melts}

\section{4-1- $\mathrm{Ni}_{77.5} \mathrm{~B}_{22.5}$}

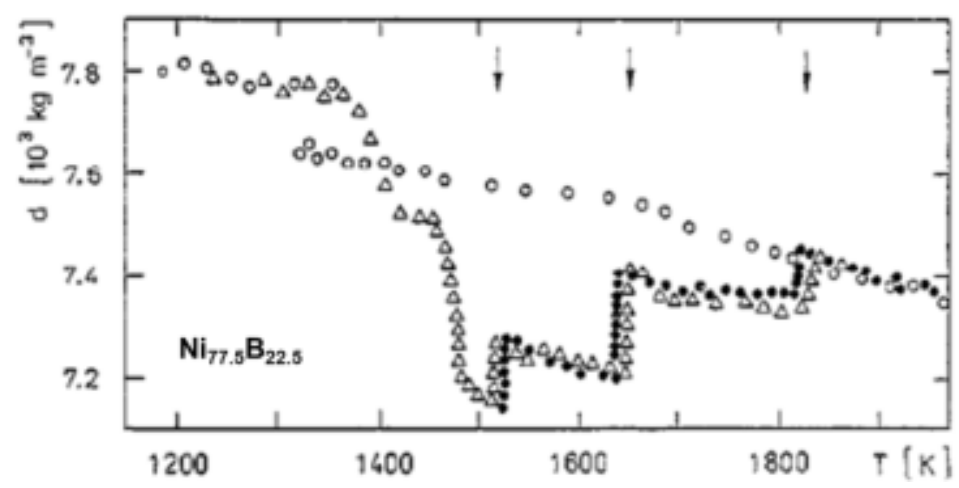

Figure 1:Reprinted from [1]: Temperature dependence of the density d of Ni-22.5\% B melt at heating after melting $(\bullet)$, subsequent cooling $(0)$, and the second heating after crystallization of the sample and repeated melting $(\Delta)$. The arrows show the 'critical' temperatures at which the density instability is observed.

Figure 1 shows the thermal variation of the density of $\mathrm{Ni}-22.5 \mathrm{at} \% \mathrm{~B}$ melt. The melting temperature is $\mathrm{T}_{\mathrm{m}}$ $=1361 \mathrm{~K}$. The sample has been exposed to an annealing time of a few hours after melting. The density increases after such annealing above $\mathrm{T}_{\mathrm{m}}$. Stable density values indicated by arrows increase with increasing temperature. These steps are viewed as due to the appearance of microdomains enriched with 
one of the components in metallic glasses. A microheterogeneous state exists in liquid alloys and is revealed by prolonged relaxation time [1]. These new liquid states are initiated in Phase 1. This alloy undergoes a transition to a glass state after hyperquenching and is transformed from Phase 2 to Phase 1 after heating through $T_{g}$ and above $T_{m}$. The glass transition temperature $T_{g}$ would have to be equal to 617 $\mathrm{K}\left(\theta_{\mathrm{g}}=-0.547\right)$ in Table 1 as expected in strong glasses having $\varepsilon_{\mathrm{gs} 0}=0.514$ and $\varepsilon_{\mathrm{ls} 0}=1.099$ [22]. The crystallization is then obtained by heating Phase 1 . Various types of Phase 1 are formed by heating, leading to exothermic latent heats at 1520 and $1645 \mathrm{~K}$ after an endothermic jump occurring at $1450 \mathrm{~K}$. The homogeneous nucleation of Phase 2 starts at $\theta_{\mathrm{n}+}=0.348, \mathrm{~T}_{\mathrm{n}+}=1835 \mathrm{~K}$ with $\varepsilon_{1 \mathrm{~s} 0} \cong 1.099$ and an endothermic latent heat equal to $0.348 \times \Delta \mathrm{H}_{\mathrm{m}}$. The density variation at $\mathrm{T}_{\mathrm{m}}$ is proportional to the latent heat of melting. The density step at $\mathrm{T}_{\mathrm{n}+}=1835 \mathrm{~K}$ is, as expected, about $35 \%$ of the density jump at $\mathrm{T}_{\mathrm{m}}$. Phase 1 is, step by step, progressively homogenized by heating.

\section{Table 1 LLPT tempe ratures de duced from thermodynamic parame ters and experiments.}

All underlined values are both experimental and calculated values. $1-T_{m}(K)$ the melting temperature of crystals, $2-T_{g}(K)$ the glass transition temperature, $3-\theta_{g}$ the reduced glass transition temperature, $4-\theta_{B r}$ the branching reduced temperature below $T_{m}$ given in (11), 5- $T_{B r}(K)$ the branching temperature below $T_{m}$, $6-\Delta \varepsilon_{l g}$ the difference $\left(\varepsilon_{l s}-\varepsilon_{g s}\right)=0$ at $T_{B r}$ below $T_{m}$, 7- $\varepsilon_{g s 0}$ the enthalpy saving coefficient $\varepsilon_{g s}$ of Phase 2 at $T_{m}, 8-\theta_{0 g}{ }^{2}$ the square of the reduced temperature where $\varepsilon_{g s}$ is equal to zero, $9-\Delta C_{p}\left(T_{g}\right)$ in J.K $K^{-} 1 / \mathrm{g}$. atom. the specific heat jump at $T_{g}$ defined in (12), 10- a the coefficient defined in (6), 11 - $\varepsilon_{l s}$ the enthalpy saving coefficient $\varepsilon_{l s}$ of Phase 1 at $T_{m}, 12-\theta_{0 m}{ }^{2}$ the square of the reduced temperature where $\varepsilon_{\text {ls }}$ is equal to zero,

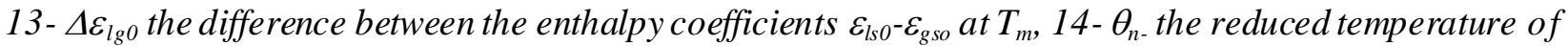
Phase 1 nucleation below $T_{m}, 15-T_{n-(}(K)$ the nucleation temperature of Phase 1 below $T_{m}, 16-\Delta \varepsilon_{l g}\left(T_{n}\right)$ the difference in (10) between $\varepsilon_{l s}$ and $\varepsilon_{g s}$ at $T_{n-\text { for }} \Delta \varepsilon=0,17$ - $\theta_{n \text { - }}^{x}$ the reduced nucleation temperature of Phase 1 below $T_{m}$ for $\Delta \varepsilon=\Delta \varepsilon_{l g}, 18-T^{e x}{ }_{n}(K)$ the nucleation temperature of Phase 1 below $T_{m}$ for $\Delta \varepsilon$ $=\Delta \varepsilon_{l g} 0,19-\Delta \varepsilon_{l g}\left(T^{e x}{ }_{n-}\right)$ the value of $\Delta \varepsilon_{l g}$ given in (10) for $\Delta \varepsilon=\Delta \varepsilon_{l g} 0$, 20- $\theta_{n+}$ the reduced nucleation temperature of Phase 2 above $T_{m}$ given in (14), 21- $T_{n+}(K)$ the nucleation temperature of Phase 2 above $T_{m}$ given in (14), 22- $\Delta \varepsilon_{l g}\left(T_{n+}\right)$ the difference $\Delta \varepsilon_{l g}$ at $T_{n+}$ given in (10) for $\Delta \varepsilon=0,23-\theta_{B r}$ the reduced branching temperature above $T_{m}$ given in (11), 24- $T_{B r}(K)$ the branching temperature above $T_{m}, 25-\Delta \varepsilon_{l g}$ $\left(T_{B r}\right)=0$ the value of $\Delta \varepsilon_{l g}$ at $T_{B r}$ given by (10) for $\Delta \varepsilon=0,26-\theta_{q 1}$ the reduced temperature of quenching leading to $\Delta \varepsilon=\Delta \varepsilon_{\text {lgo }}$ frozen in Phase 2 and of a new LLPT, 27- $T_{q 1}(K)$ the temperature of Phase 2 quenching and of a new LLPT, 28- $\Delta \varepsilon_{l g}\left(T_{q 1}\right)$ the value of $\Delta \varepsilon_{l g}=-\Delta \varepsilon_{l g}$ at $T_{q 1}$ for $\Delta \varepsilon=0$ and equal to zero in (10) for $\Delta \varepsilon=\Delta \varepsilon_{l g 0}, 29$ - $\theta_{q 2}$ the reduced temperature of quenching leading to $\Delta \varepsilon=\Delta \varepsilon_{l g 0}$ frozen in Phase 2 , 30- $T_{q 2}(K)$ the temperature of quenching of Phase 2 where $\Delta \varepsilon_{l g}=-2 \times \Delta \varepsilon_{l g}, 31-\Delta \varepsilon_{l g}\left(T_{q 2}\right)$ the value of $\Delta \varepsilon_{l g}=-2 \times \Delta \varepsilon_{l g 0}$ at $T_{q 2}$ in (10) for $\Delta \varepsilon=0$ and equal to zerofor $\Delta \varepsilon=2 \times \Delta \varepsilon_{l g 0}$, 32- $T_{t s}(K)$ the temperature above which the melt becomes a true solution.

$\begin{array}{lllllll} & & \mathbf{N i}_{77.5} \mathbf{B}_{\mathbf{2 2 5}} & (\mathbf{F e} . .)_{\mathbf{9}} \mathbf{N b}_{\mathbf{4}} & \mathbf{V i t 1 0 5} & \mathbf{V i t 1 0 6} & \text { Vit1 } \\ 1 & \mathbf{T}_{\mathbf{m}} & 1361 & 1410 & 1072 & 1115 & 965 \\ 2 & \mathbf{T}_{\mathbf{g}} & 617 & 863 & 675 & 682 & 625 \\ 3 & \boldsymbol{\theta}_{\mathbf{g}} & -0.547 & -0.388 & -0.370 & -0.388 & -0.352 \\ 4 & \boldsymbol{\theta}_{\mathbf{B r}} & -0.547 & -0.317 & -0.302 & -0.304 & -0.254 \\ 5 & \mathbf{T}_{\mathbf{B r}} & 617 & 963 & 748 & 776 & 720 \\ 6 & \boldsymbol{\Delta} \boldsymbol{\varepsilon}_{\mathbf{l g}}\left(\mathbf{T}_{\mathbf{B r}}\right) & 0 & 0 & 0 & 0 & 0 \\ 7 & \boldsymbol{\varepsilon}_{\mathbf{g s} \mathbf{0}} & 0.514 & 1.418 & 1.444 & 1.417 & 1.472 \\ 8 & \boldsymbol{\theta}_{\mathbf{g g}}{ }^{2} & 1 & 0.367 & 0.357 & 0.367 & 0.346 \\ 9 & \boldsymbol{\Delta} \mathbf{C}_{\mathbf{p}}\left(\mathbf{T}_{\mathbf{g}}\right) & & 10 & & 16.0 & 23.6 \\ 10 & \mathbf{a} & & 1 & 1 & 0.918 & 0.780\end{array}$




\begin{tabular}{|c|c|c|c|c|c|c|}
\hline 11 & $\varepsilon_{\mathrm{ls} 0}$ & 1.099 & 1.612 & 1.63 & 1.644 & 1.725 \\
\hline 12 & $\theta_{0 \mathrm{~m}^{2}}$ & 0.444 & 0.278 & 0.268 & 0.260 & 0.211 \\
\hline 13 & $\Delta \varepsilon_{\lg 0}$ & 0.585 & 0.194 & 0.185 & 0.227 & 0.254 \\
\hline 14 & $\theta_{\mathrm{n}-}$ & -0.666 & -0.259 & -0.247 & $\underline{-0.238}$ & $\underline{-0.183}$ \\
\hline 5 & $\mathbf{T}_{\mathbf{n}-}$ & 454 & 1045 & 807 & $\underline{850}$ & 788 \\
\hline 16 & $\Delta \varepsilon_{\lg }\left(T_{n-}\right)$ & -0.285 & 0.065 & 0.062 & 0.174 & 0.122 \\
\hline 17 & $\theta^{\mathrm{ex}}{ }_{\mathrm{n}-}$ & -0.318 & -0.164 & -0.157 & $\underline{-0.238}$ & -0.103 \\
\hline 18 & $T^{e x}{ }_{n-}$ & 929 & 1179 & 904 & 952 & 865 \\
\hline 19 & $\Delta \varepsilon_{\lg }\left(T^{e x}{ }_{n-}\right)$ & 0.388 & 0.142 & 0.157 & 0.174 & 0.212 \\
\hline 20 & $\theta_{n+}$ & $\underline{0.348}$ & $\underline{0.150}$ & $\underline{0.143}$ & 0.162 & $\underline{0.157}$ \\
\hline 21 & $T_{n+}$ & 1835 & 1622 & 1226 & 1296 & 1116 \\
\hline 22 & $\Delta \varepsilon_{\lg }\left(\mathbf{T}_{\mathbf{n}+}\right)$ & $\underline{0.348}$ & 0.150 & 0.143 & 0.162 & $\underline{0.157}$ \\
\hline 23 & $\theta_{\mathrm{Br}}$ & 0.547 & 0.317 & 0.302 & $\underline{0.304}$ & 0.254 \\
\hline 24 & $\mathbf{T}_{\mathrm{Br}}$ & 2105 & 1857 & 1396 & 1454 & $\underline{1210}$ \\
\hline 25 & $\Delta \varepsilon_{\lg }\left(T_{B r}\right)$ & 0 & 0 & 0 & 0 & $\underline{\mathbf{0}}$ \\
\hline 26 & $\theta_{q 1}$ & 0.749 & 0.448 & 0.428 & 0.430 & 0.359 \\
\hline 27 & $\mathbf{T}_{\mathbf{q} 1}$ & 2380 & 2042 & 1530 & 1594 & 1312 \\
\hline 28 & $\Delta \varepsilon_{\lg }\left(\mathbf{T}_{\mathrm{q} 1}\right)$ & $-\Delta \varepsilon_{\lg 0}$ & $-\Delta \varepsilon_{\lg 0}$ & $-\Delta \varepsilon_{\lg 0}$ & $-\Delta \varepsilon_{\lg 0}$ & $-\Delta \varepsilon_{\lg 0}$ \\
\hline 29 & $\theta_{\mathrm{q} 2}$ & 0.907 & 0.549 & 0.524 & 0.526 & 0.440 \\
\hline 30 & $\mathbf{T}_{\mathbf{q} 2}$ & 2596 & 2184 & 1633 & 1702 & 1390 \\
\hline 31 & $\Delta \varepsilon_{\lg }\left(\mathbf{T}_{\mathbf{q} 2}\right)$ & $-2 \Delta \varepsilon_{\lg 0}$ & $-2 \Delta \varepsilon_{\lg 0}$ & $-2 \Delta \varepsilon_{\lg 0}$ & $-2 \Delta \varepsilon_{\lg 0}$ & $-2 \Delta \varepsilon_{\lg 0}$ \\
\hline 32 & $\mathbf{T}_{\mathrm{ts}}$ & 2722 & 2264 & 1712 & 1790 & 1532 \\
\hline
\end{tabular}

A branching temperature is observed at $T_{n+}=1835 \mathrm{~K} \mathrm{[1]} \mathrm{instead} \mathrm{of} \mathrm{the} \mathrm{predicted} \mathrm{value} \mathrm{of} 2105 \mathrm{~K}$ given in Table 1. The thermal properties of Phase 1 are reversible up to $\mathrm{T}_{\mathrm{Br}}$, where a new branching temperature is expected with a formation of Phase 2. Phase 2 clusters would disappear at $\mathrm{T}_{\mathrm{ts}}=2722 \mathrm{~K}$.

\section{2- $\left(\mathrm{Fe}_{71.2} \mathrm{~B}_{24} \mathrm{Y}_{4.8}\right){ }_{96} \mathrm{Nb}_{4}$}

Amorphous alloys of $\left(\mathrm{Fe}_{71.2} \mathrm{~B}_{24} \mathrm{Y}_{4.8}\right)_{96} \mathrm{Nb}_{4}$ have been prepared by fast cooling of the melt from the temperature range of $1573-1773 \mathrm{~K}$. The melting temperature is $1410 \mathrm{~K}$ and the glass transition temperature $863 \mathrm{~K}$ [6]. All properties of this melt are described in Table 1 using (16-20) and assuming that scaling laws are followed and consequently a $=1$. All LLPT temperatures as well as the fraction of melting heat associated with these first-order transitions are predicted. An endothermic heat has been observed at 1615-1650 K (6) and corresponds to the nucleation temperature $T_{n+}=1622 \mathrm{~K}$ of Phase 2 in Phase 1 predicted in (18) with $\theta_{\mathrm{g}}=-0.38794$. The latent heat would have to be equal to $15 \%$ of the melting heat.

\section{3- $\mathrm{Zr}_{52.5} \mathrm{Cu}_{17.9} \mathrm{Ni}_{14.6} \mathrm{Al}_{10} \mathrm{Ti}_{5}(\mathrm{Vit} 105)$}

The glass transition temperature is $T_{g}=675 \mathrm{~K}$ and the melting temperature $T_{m}=1072 \mathrm{~K}$ ( $\mathrm{T}$ solidus). A very precise overheating threshold has been observed at $1225 \mathrm{~K}$ on the supercooling rate of Vit 105 , as shown in Figure 2 [32]. There are two nucleation temperatures of the same crystalline phase in liquid Phase 1 and liquid Phase 2. The model predicts $T_{n+}=1225 \mathrm{~K}$ for the nucleation of Phase 2 with (18), $\theta_{\mathrm{g}}=-0.37034$ assuming $\mathrm{a}=1$. All parameters given in Table 1 are calculated from (16-20). Using a specific heat jump 
measurement of $\Delta \mathrm{C}_{\mathrm{p}}\left(\mathrm{T}_{\mathrm{g}}\right)=17.1 \mathrm{~J} / \mathrm{at} . \mathrm{g}$ (33), a $=0.86$, the predicted temperature $\mathrm{T}_{\mathrm{n}+}$ would be equal to 1243 $\mathrm{K}(+1.47 \%)$.

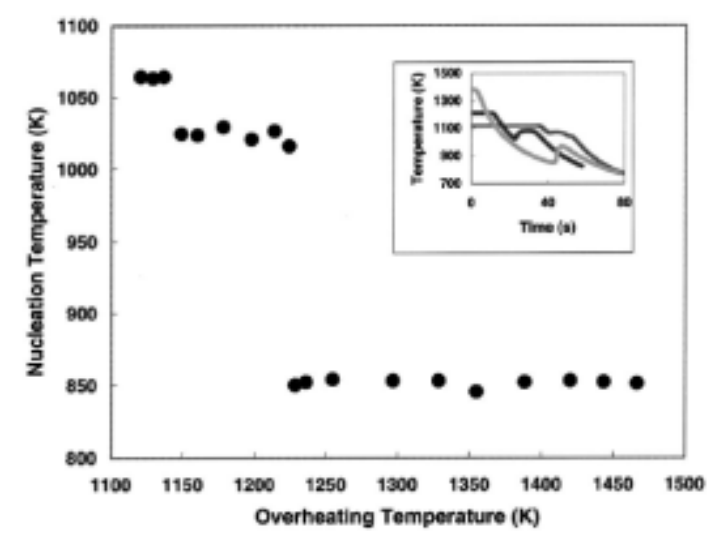

Figure 2: Reprinted from [32], (Copyright 2004 American Institute of Physics). Nucleation temperature vs overheating temperature for $\mathrm{Zr}_{52.5} \mathrm{Cu}_{17.9} \mathrm{Ni}_{14.6} \mathrm{Al}_{10} \mathrm{Ti}_{5}$ (Vit105). The cooling curves obtained from different levels of overheating are shown in the inset, in which the temperature at time $t=0 \mathrm{~s}$ shows the level of overheating.

\section{4- $\mathrm{Zr}_{57} \mathrm{Cu}_{15.4} \mathrm{Ni}_{12.6} \mathrm{Al}_{10} \mathrm{Nb}_{5}$ (Vit 106)}

Two LLPT have already been obtained. The first one around $1450 \mathrm{~K}$ observes the specific volume as a function of temperature [7] below this branching temperature, the second one at 950-1000 K measures a structural crossover [8]. These two events correspond to $\mathrm{T}^{\mathrm{ex}}=952 \mathrm{~K}$ and $\mathrm{T}_{\mathrm{Br}}=1454 \mathrm{~K}$ in Table 1. The calculation is made using a homogeneous nucleation temperature $\mathrm{T}_{\mathrm{n} 1-}$ of crystallization in Phase 1 equal to $850 \mathrm{~K}$, as observed by [8]. The corresponding values of a and $\Delta \mathrm{C}_{\mathrm{p}}\left(\mathrm{T}_{\mathrm{g}}\right)$ are 0.918 and $16 \mathrm{~J} / \mathrm{g}$.atom respectively. The structural crossover is calculated at $952 \mathrm{~K}$ because an overheating above $1600 \mathrm{~K}$ followed by rapid cooling has been used and results in freezing an enthalpy excess of $\left(\varepsilon_{1 \mathrm{~s} 0^{-}}\right.$ $\left.\varepsilon_{\mathrm{gs} 0}\right) \times \Delta \mathrm{H}_{\mathrm{m}}=0.2265 \times \Delta \mathrm{H}_{\mathrm{m}}$

\section{4-5- $\mathrm{Zr}_{41.2} \mathrm{Ti}_{13.8} \mathrm{Cu}_{12.5} \mathrm{Ni}_{10} \mathrm{Be}_{22.5}(\mathrm{Vit} 1)$}

The glass transition of this melt is $T_{g}=625 \mathrm{~K}$ and its melting temperature $965 \mathrm{~K}$ is chosen in the middle of the liquidus-solidus temperature window [34]. For the specific heat jump $\Delta \mathrm{C}_{\mathrm{p}}\left(\mathrm{T}_{\mathrm{g}}\right)=23.6 \mathrm{~J} / \mathrm{g}$.atom of this fragile glass and $\Delta \mathrm{H}_{\mathrm{m}}=8200 \mathrm{~J} / \mathrm{g}$.atom, the value of a is 0.78 . The LLPT temperatures are deduced and given in Table 1. The thermal variation of $\Delta \varepsilon_{\lg }$ given in (10) without frozen enthalpy $(\Delta \varepsilon=0)$ is represented in Figure 3. Two branching temperatures are expected for $\Delta \varepsilon_{1 \mathrm{~g}}=0$, the first one at $720 \mathrm{~K}$ and the second one at $1210 \mathrm{~K}$. Phase 1 is the most stable phase between 720 and $1210 \mathrm{~K}$. Phase 2 is the most stable phase below $720 \mathrm{~K}$ and above $1210 \mathrm{~K}$. 


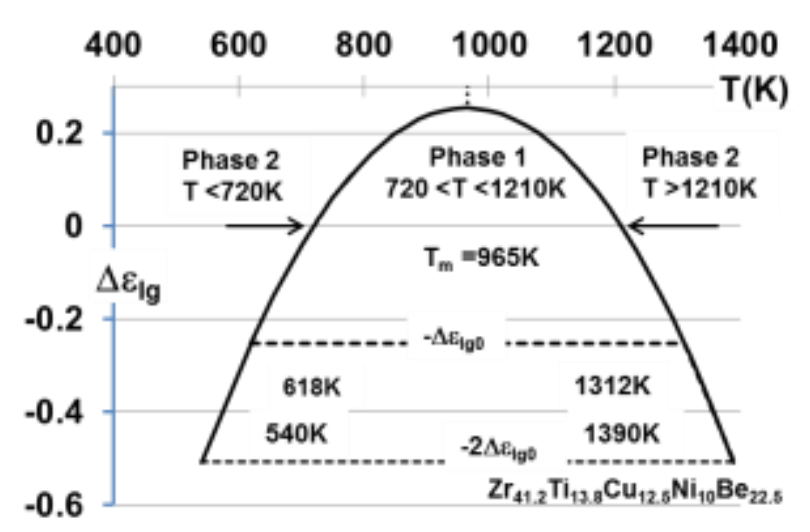

Figure 3: Thermal variation of $\Delta \varepsilon_{\text {lg }}$ given by (10) for $\Delta \varepsilon=0$. The most stable phase between 720 and 1210 $\mathrm{K}$ is Phase 1 and below $720 \mathrm{~K}$ and above $1210 \mathrm{~K}$ Phase 2 . At the temperature $T_{q 1}=1312 \mathrm{~K}$, Phase 1 being not stable has an enthalpy excess of $\Delta \varepsilon_{l g 0} \times \Delta H_{m}=\left(\varepsilon_{l s 0^{-}}-\varepsilon_{g s 0}\right) \times \Delta H_{m}$ as compared with Phase 2 . Cooling the sample in Phase 1 instead of Phase 2 fromabove $T_{q 1}$ down to $T_{q 1}$ would lead to a LLPTfrom Phase 1 to Phase 2 because $\Delta \varepsilon_{l g}$ becomes equal to zero including $\Delta \varepsilon=\Delta \varepsilon_{l g}$ in (10). At the temperature $T_{q 2}=1390 \mathrm{~K}$, Phase 1 has an enthalpy excess of $2 \times\left(\varepsilon_{l s 0^{-}} \varepsilon_{g s}\right) \times \Delta H_{m}$.

The homogeneous nucleation temperatures of Phase 2 are $T_{g}=625 \mathrm{~K}$ and $T_{n+}=1116 \mathrm{~K}$. The branching temperature $\mathrm{T}_{\mathrm{Br}}$ has been observed at $1225 \mathrm{~K}$ on the viscosity and predicted at $1210 \mathrm{~K}$. Figure 4 represents the branching of this quantity [5]. The viscosity has Newtonian behavior when the phase is the most stable.

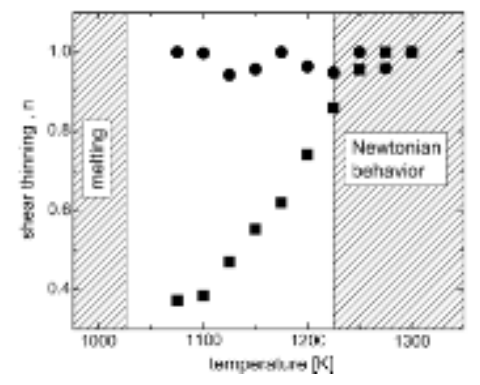

Figure 4: Reprinted from [5]. Non-Newtonian behavior vs temperature of initially amorphous Vit1 for a first ( $\square$ ) and a second ( $(0)$ temperature scan. After melting, the alloy exhibits a pronounced shear thinning dependence of the viscosity that decreases with increasing temperature. Above $1225 \mathrm{~K}$, there is no longer a shear rate dependence of the viscosity. After cooling back to $1075 \mathrm{~K}$ and isothermal holding for $1 \mathrm{~h}$, the material is still Newtonian over the investigated temperature range.

The enthalpy coefficients $-\varepsilon_{\mathrm{ls}}$ of Phase 1 and $-\varepsilon_{\mathrm{gs}}$ of Phase 2 given by (2) and (3) are represented in Figure 5 between $\mathrm{T}_{\mathrm{g}}=625 \mathrm{~K}$ and $1225 \mathrm{~K}$, including the latent heat coefficients equal to $\left(\varepsilon_{1 \mathrm{~s}}-\varepsilon_{\mathrm{gs}}\right)$ at $\mathrm{T}_{\mathrm{n}-}=788 \mathrm{~K}$ and $\mathrm{T}_{\mathrm{n}+}=1116 \mathrm{~K}$. Two LLPT have been observed at 760-830 K [35] and 1100-1200 K [4] as well as latent heats equal to about $900 \mathrm{~J} / \mathrm{g}$.atom [34] and $1100 \mathrm{~J} / \mathrm{g}$. atom respectively. The $\left(\varepsilon_{1 \mathrm{~s}}-\varepsilon_{\mathrm{gs}}\right)=0.122 * 8200=1000$ $\mathrm{J} / \mathrm{g}$.atom at $788 \mathrm{~K}$ and $0.157 * 8200=1287 \mathrm{~J} / \mathrm{g}$.atom are predicted in Table 1 . The latent heat at $788 \mathrm{~K}$ is exothermic and corresponds to a transition from Phase 2 to Phase 1 . That at $1116 \mathrm{~K}$ is endothermic and occurs at the nucleation temperature of Phase 2 in Phase 1 . These two temperatures are not symmetrical as 
compared to $T_{m}=965 \mathrm{~K}$. The latent heat at $T_{n+}$ is always larger than that at $T_{n-.}$ Consequently, there is always a fraction of enthalpy which is not recovered below $T_{n}$ in Phase 1.

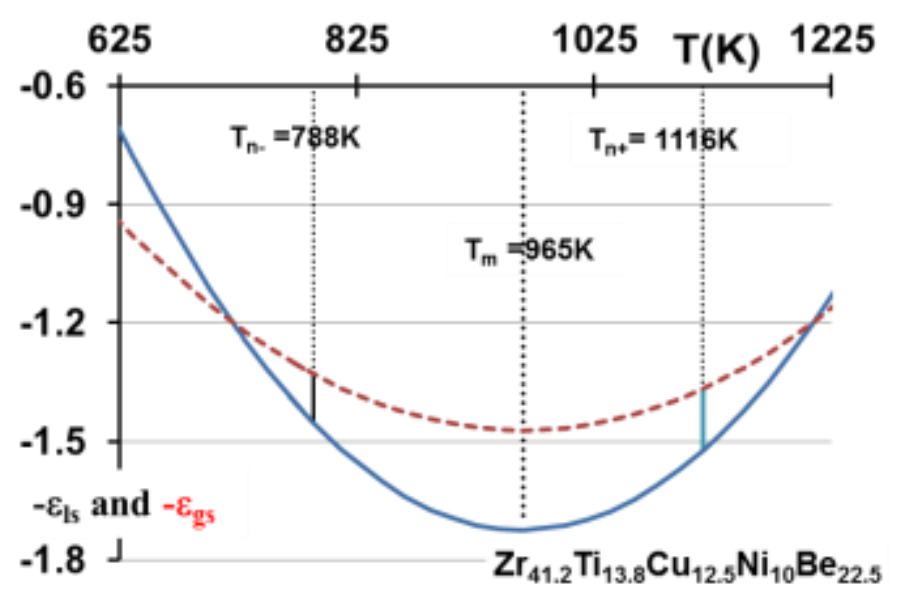

Figure 5: The enthalpy coefficients $-\varepsilon_{l s}$ of Phase 1 (solid curve) and- $\varepsilon_{g s}$ of Phase 2 (dashed curve) vs temperature from $625 \mathrm{~K}$ up to $1225 \mathrm{~K}$. Two LLPT are represented at $788 \mathrm{~K}$ and $1116 \mathrm{~K}$. The first one is the homogeneous nucleation temperature of Phase 1 in Phase 2. The second one is that of Phase 2 in Phase 1.

The enthalpy coefficients $-\varepsilon_{\mathrm{ls}}$ of Phase 1 and $-\varepsilon_{\mathrm{gs}}$ of Phase 2 given by (2) and (3) are represented in Figure 6 between $\mathrm{T}_{\mathrm{m}}=965 \mathrm{~K}$ and $\mathrm{T}_{\mathrm{ts}}=1532 \mathrm{~K}$, including latent heat coefficients equal to $\left(\varepsilon_{1 \mathrm{~s} 0}-\varepsilon_{\mathrm{g} 50}\right)$ at $\mathrm{T}_{\mathrm{q} 1}=1312 \mathrm{~K}$ and $2 \times\left(\varepsilon_{1 \mathrm{~s} 0}-\varepsilon_{\mathrm{gs} 0}\right)$ at $\mathrm{T}_{\mathrm{q} 2}=1390 \mathrm{~K}$. A new LLPT is expected at $\mathrm{T}_{\mathrm{q} 1}=1312 \mathrm{~K}$ after heating Phase 1 above this temperature and cooling it down to $1312 \mathrm{~K}$. The temperature $\mathrm{T}_{\mathrm{q} 2}=1390 \mathrm{~K}$ is indicated because it determines the upper limit of enthalpy excess leading to an ultrastable glass state after hyperquenching and liquid deposition on a substrate cooled below $\mathrm{T}_{\mathrm{g}}[26]$.

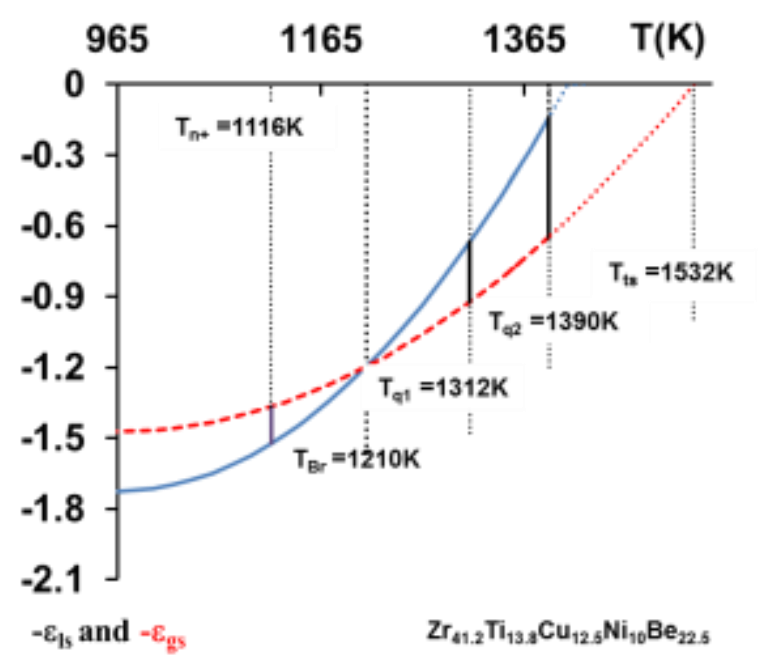

Figure 6: Enthalpy coefficients $-\varepsilon_{l s}$ (solid curve) and- $\varepsilon_{g s}$ (dashed curve) of Phase 1 and Phase 2 given by (2) and (3) vs temperature from $T_{m}$ up to the true solution temperature $T_{t s}=1532 \mathrm{~K}$. A new LLPT is expected at $T_{q 1}=1312 \mathrm{~K}$. 


\section{5- Thermal paths leading to Phase 1 and Phase 2}

Slow cooling below $\mathrm{T}_{\mathrm{m}}$ of Phase 1 and Phase 2 leads to only one crystal phase. New melting of crystals would have to stabilize Phase 1 above $T_{m}$ instead of Phase 2.

Quenching Phase 1 from a temperature less than $T_{n+}$ does not induce a LLPT at $T_{n-.}$ A tendency to crystallization can exist at $\mathrm{T}_{\mathrm{n}}$ - because this temperature is also the homogeneous nucleation temperature of growth nuclei of crystals in Phase 1.

Heating Phase 1 up to $\mathrm{T}_{\mathrm{n}+}$ and above leads to Phase 2 through a LLPT [4,32]. A rapid cooling induces a LLPT at $T_{n}$ - which transforms Phase 2 into Phase 1 above $T_{g}$. Phase 1 is transformed into Phase 2 at $T_{g}$.

A rapid heating above $\mathrm{T}_{\mathrm{q} 1}$ of Phase 2 followed by rapid cooling freezes an enthalpy excess in Phase 2 equal to $\left(\varepsilon_{1 \mathrm{~s} 0}-\varepsilon_{\mathrm{gs} 0}\right)$ and produces a LLPT at $\mathrm{T}^{\mathrm{ex}}{ }_{\mathrm{n} \text { - }}$, the nucleation temperature of Phase 1 in Phase 2 [8]. A continuous cooling below $T^{\mathrm{ex}}{ }_{\mathrm{n} \text { - }}$ of Phase 1 leads to $\mathrm{T}_{\mathrm{n}}$ - corresponding to the homogeneous nucleation temperature of some growth nuclei of crystals in Phase 1 . Phase 1 is transformed into Phase 2 at $T_{g}$. With a new heating above $T_{g}$, Phase 2 is transformed into Phase 1 at $T_{g}$.

A rapid heating from the glass state up to $T_{n+}$ induces a LLPT at $T_{n+}$ transforming Phase 1 into Phase 2 [4].

\section{6- Time dependence of the glass transition temperature $T_{g}$}

The glass transition temperature $T_{g}$ depends on the heating and cooling rates, even if $T_{g}$ has a well-defined value at low rates [36]. Using (5), the glass transition temperature of Vit1 is plotted in Figure 7 as a function of the enthalpy excess coefficient $\Delta \varepsilon$ which has not been recovered in Phase 1 . The $\mathrm{T}_{\mathrm{g}}$ can be increased from 625 to $720 \mathrm{~K}$ with $\Delta \varepsilon$ varying from zero to 0.0411 . The temperature $720 \mathrm{~K}$ corresponds to the branching temperature close to $T_{g}$. The $T_{g}$ of $V$ it 1 increases up to $675 \mathrm{~K}$ by varying the heating rate from $0.0167 \mathrm{~K} / \mathrm{s}$ to $6.66 \mathrm{~K} / \mathrm{s}$ [34]. This increase corresponds to a value of $\Delta \varepsilon=0.0115$. The enthalpy excess coefficient which has not been recovered at $T_{n}$ is equal to $(0.157-0.122)=0.035$ as shown in Table 1 . Then, $\mathrm{T}_{\mathrm{g}}$ can still be increased with the heating rate. The time dependence of $\mathrm{T}_{\mathrm{g}}$ shows that the residual enthalpy excess is relaxed in Phase 2 below $T_{g}$ instead of Phase 1.

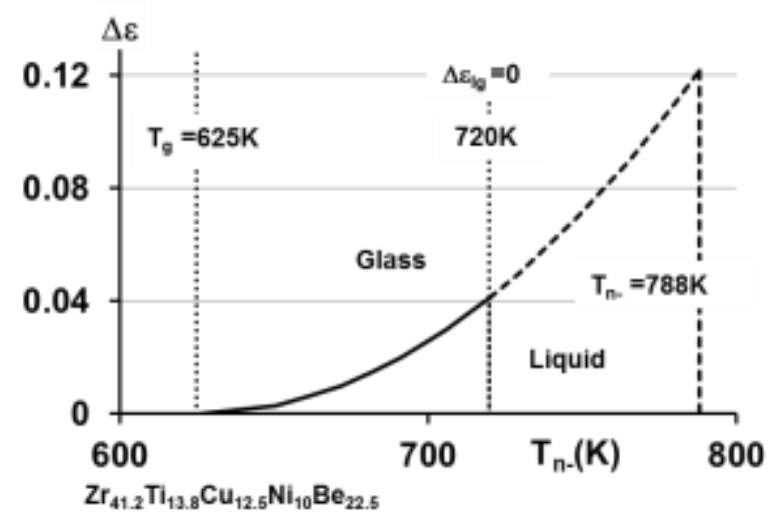


Figure 7: The enthalpy excess coefficient $\Delta \varepsilon v$ s the glass transition up to $720 \mathrm{~K}$ as given in (5). Above 720 $K$, there are thermodynamic transitions associated with the presence of an enthalpy excess at $T_{n-}=788 \mathrm{~K}$ and $T_{n-x}=865 \mathrm{~K}$. The other values of $\Delta \varepsilon$ above $720 \mathrm{~K}$ are not used. In all fragile glasses, $T_{g}$ is strongly dependent on small values of $\Delta \varepsilon$.

\section{7- Application to all liquids}

This model can be applied to all liquids, even if they have never been transformed by quenching into glasses and if there is no proof of the existence of such LLPT in non-metallic glasses. The case of eutectic $\left(\mathrm{Al}_{82.8}-\mathrm{Cu}_{17.2}\right)$ is examined [37]. The melting temperature $\mathrm{T}_{\mathrm{m}}$ is equal to $821.3 \mathrm{~K}$ and the formation temperature of Phase 2 is equal to $1603 \mathrm{~K}$ below $\mathrm{T}_{0 \mathrm{~g}}=2 \times \mathrm{T}_{\mathrm{m}}=1642.6 \mathrm{~K}\left(\theta_{0 \mathrm{~g}}=-1\right.$ as in pure liquid elements) [24]. The value of $\varepsilon_{\mathrm{gs}}$ in Phase 2 at $\mathrm{T}=1603 \mathrm{~K}(\theta=0.952)$ is $0.217 \times\left(1-\theta^{2}\right)=0.02$. The highest condensation temperature of Phase 2 at $1603 \mathrm{~K}$ is accompanied by a branching of thermal properties because Phase 1 can be rapidly heated from $\mathrm{T}_{\mathrm{m}}$ up to $1603 \mathrm{~K}$ [37]. There is slope breaking at $1368 \mathrm{~K}$ corresponding to the melting of Phase 1 at $\theta=-2 / 3$ and the melt entrance into a solution without Phase 1 . Phase 2 cluster formation occurs between 1603 and $1642.6 \mathrm{~K}$. Phase 2 is induced at the homogeneous nucleation temperature determined by $\varepsilon_{\mathrm{gs}}=0.02$.

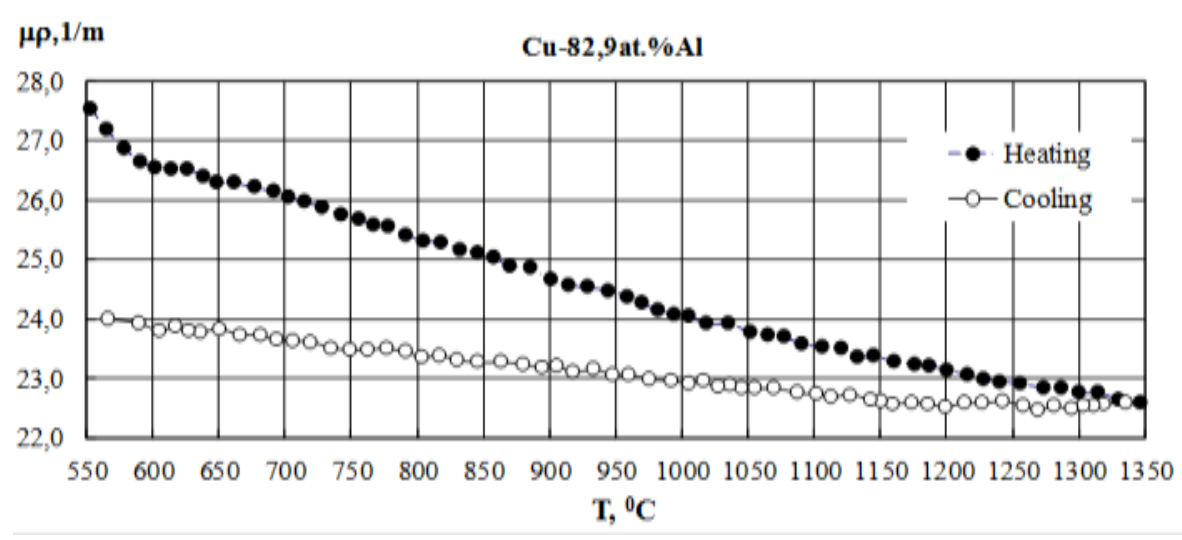

Figure 8: Reprinted from [37] with permission of Springer. Temperature dependences of $\rho \mu(\rho-$ density of the melt, $\mu$ - its gamma-quanta mass coefficient of absorption) for Al-Cu17.2 at\% alloy.

\section{Conclusions}

Metallic glass and glass-forming melt properties are the result of competition between two liquid phases called Phase 1 and Phase 2 having an enthalpy difference varying with the square of the reduced temperature $\theta=\left(T-T_{m}\right) / T_{m}$. The transitions between these phases are governed by the classical equation of nucleation completed by this enthalpy saving. The liquid-to-liquid phase transitions (LLPT) of glassforming melts are universal when they obey scaling laws and are determined knowing only $\mathrm{T}_{\mathrm{g}}$ and the melting temperature $T_{m}$ of crystallized phase. These glasses have a specific heat jump $\Delta \mathrm{C}_{\mathrm{p}}\left(\mathrm{T}_{\mathrm{g}}\right)$ $=1.5 \times \Delta \mathrm{H}_{\mathrm{m}} / \mathrm{T}_{\mathrm{m}}$ from Phase 2 to Phase 1 , heating the glass through $\mathrm{T}_{\mathrm{g}}$, and a homogeneous reduced nucleation temperature of Phase 1 equal to $\theta_{\mathrm{g}} / 1.5$ observed by cooling. The homogeneous nucleation temperature of Phase 1 in Phase 2 in other glass-forming melts with a specific heat jump $\Delta \mathrm{C}_{\mathrm{p}}\left(\mathrm{T}_{\mathrm{g}}\right)$ larger than $1.5 \times \Delta \mathrm{S}_{\mathrm{m}}$ is equal to $\mathrm{a} \times \theta_{\mathrm{g}} / 1.5$, where a is deduced from $\Delta \mathrm{C}_{\mathrm{p}}\left(\mathrm{T}_{\mathrm{g}}\right)$. 
Four LLPT are predicted. Three have been observed up to now at the predicted temperatures in several glass-forming melts. Two nucleation temperatures of crystallized phases depending on supercooling of Phase 1 and Phase 2 were obtained in Vit105. The junction temperatures of Phase 1 and Phase 2 correspond to temperatures above which the thermal properties become reversible.

The LLPT of melts which have not been recognized, up to now, as glass-forming melts, can be predicted, assuming that their glass phase is analogous to that of strong glasses. The disappearance of any Phase 2 at $\mathrm{T}_{\mathrm{ts}}$ in the melt true solution is determined. The Phase 2 formation temperature occurs at a branching temperature of thermal properties not far below $\mathrm{T}_{\mathrm{ts}}$.

\section{References}

[1] P.S. Popel, V.E. Sidorov. Mater. Sci. Eng. A226-228 (1997) 237.

[2] P.S. Popel, V.V. Majekev, Zh. Phys. Chim. (USSR), 64 (1990) 568.

[3]. P.S. Popel, N.Y. Konstantinova, J. Phys. Conf. Series. 2008, Vol. 98, p. 062022.

[4] S. Wei, F. Yang, J. Bednarcik, I. Kaban, O. Shuleshova, A. Meyer, \& R. Busch, Nature Comm. 4 (2013) 2083.

[5] C. Way, P. Wadhwa, and R. Busch, Acta Mater. 55 (2007) 2977.

[6] Q. Hu, H.C. Sheng, M.W. Fu, X.R. Zeng. J. Mater. Sci. 49 (2014) 6900.

[7] J.J.Z. Li, W.K. Rhim, C.P. Kim, K. Samwer, W.L. Johnson, Acta Mater. 59 (2011) 2166.

[8] S. Lan, M. Blodgett, K.F. Kelton, J.L. Ma, J. Fan, and X.L. Wang, Appl. Phys. Lett. 108 (2016) 211907.

[9] S.Wei, I. Gallino, R. Busch, and C.A. Angell, Nature Phys. 7 (2011) 178.

[10] H. Tanaka, R. Kurita, H. Mataki, Phys. Rev. Lett. 92 (2004) 025701.

[11] T.R. Kirkpatrick, D. Thirumalai, Phys. Rev. A. 31 (1985) 939.

[12] J. Souletie, J. Phys. France 51 (1990) 883.

[13] L. Berthier et al, Science 310 ( 2005) 1797.

[14] C.A. Angell, P.G. Wolynes, and V. Lubchenko [Eds]. Structural glasses and supercooled liquids. Wiley and Sons, Hoboken, NY, (2012) 237-278.

[15] M.I. Ojovan, K.P. Travis, R.J. Hand, J. Phys. Condens. Matter 19 (2007) 415107.

[16] M.I. Ojovan, J. Non-Cryst. Sol. 382 (2013) 79.

[17] J.F. Stanzione III, K.E. Strawhecker, R.P. Wool, J. Non-Cryst. Sol. 357 (2011) 311.

[18] R.P. Wool, J. Polymer. Sci. B 46 (2008) 2765. 
[19] R.P. Wool, A. Campanella, J. Polymer Phys. B 47 (2009) 2578.

[20] D.S. Sanditov, J. Non-cryst. Sol. 385 (2014) 148.

[21] F. Albert et al., Science 352 (2016) 1308.

[22] R.F. Tournier, Physica B 454 (2014) 271.

[23] R.F. Tournier, Phys. B Condens. Matter 392 (2007) 79.

[24] R.F.Tournier, Chem. Phys. Lett. 651 (2016) 198.

[25] R.F. Tournier, J. Bossy, Chem. Phys. Lett. 658 (2016) 282.

[26] R.F. Tournier, Chem. Phys. Lett. 641 (2015) 9.

[27] D. Turnbull, Chem. Phys. 20 (1952) 411.

[28] R.F. Tournier, Revue de Metall. 109 (2012) 27.

[29] R.F. Tournier, Sci. Technol. Adv. Mater. 10 (2009) 014607.

[30] R.F. Tournier, Materials 4 (2011) 869.

[31] C-Y. Liu, J. He, R. Keunings, C. Bailly, Macromolecules 39 (2006) 8867.

[32] S. Mukherjee, Z. Zhou, J. Schroers,W.L. Johnson, and W.K. Rhim, Appl. Phys. Lett. 84 (2004) 5010.

[33] S.C. Glade, R. Busch, D.S. Lee, W.L. Johnson, R.K. Wunderlich, H.J. Fecht, J. Appl. Phys. 87 (2000) 7242.

[34] R. Busch, Y.J. Kim, W.L. Johnson, J. Appl. Phys. 77 (1995) 4039.

[35] K. Ohsaka et al., Appl. Phys. Lett. 70 (1997) 726.

[36] Z. Cernosek, J. Holubova, E. Cernoskova, M. Liska, Adv. Mat. 4 (2002) 489.

[37] A.R. Kurochkin, A.V. Borisenko, P.S. Popel, D.A. Yagodin, A.V. Okhapkin, High Temp. 51 (2013) 197. 